\title{
DISEÑO DE UN PROTOTIPO DE SISTEMA DE REALIDAD VIRTUAL INMERSIVO SIMPLIFICADO
}

\author{
PROTOTYPE DESIGN OF A VIRTUAL IMMERSIVE SIMPLIFY SYSTEM
}

Christian David, Quintero Guerrero

Ing. Multimedia, Joven Investigador, Universidad Militar Nueva Granada, Facultad de Ingeniería, Grupo de Investigación en Multimedia, Bogotá, Colombia, u1200100@umng.edu.co

Eduardo Leonardo, Sierra Ballen

Ing. Sistemas, M. Eng (c) - Ing. de Sistemas y Computación, Profesor Auxiliar, Facultad de Ingeniería, Universidad Militar Nueva Granada, Grupo de Investigación en Multimedia, Bogotá, Colombia, elsierra@umng.edu.co

Wilson Javier, Sarmiento Manrique

Ing. Sistemas, M. Eng - Ing. de Sistemas y Computación, Profesor Auxiliar, Facultad de Ingeniería, Universidad Militar Nueva Granada, Grupo de Investigación en Multimedia, Bogotá, Colombia, wjaviers@umng.edu.co

Fecha de recepción: 7 de abril de 2008.

Fecha de aprobación: 16 de junio de 2008.

\section{RESUMEN}

Este artículo presenta el diseño y construcción de un prototipo de bajo costo de un sistema inmersión virtual de dos pantallas. Se describen los detalles más importantes de la implementación y se muestran las ventajas y problemas de diferentes arquitecturas posibles para el montaje físico. Se construyeron tres prototipos, uno de mesa y dos de tamaño real, sobre los cuales se realizaron diferentes pruebas con animaciones creadas en un software de modelado y animación 3D.

Palabras claves: Sistemas inmersivos, CAVE, tubería de vista, realidad virtual.

\begin{abstract}
This paper introduces the development of a low cost prototype of a virtual immersion system with two screens. The main implementation details are presented, so that the advantages
\end{abstract}


and drawbacks from many physic assembly architectures. Three prototypes were constructed, one table system and the other two at real size. Thus, different tests were implemented with animations, which were created using a 3D modeling and animation software.

Key words: Immersive systems, CAVE, view pipeline, virtual reality.

\section{INTRODUCCIÓN}

En los últimos años, el manejo de grandes volúmenes de información cada vez más compleja ha motivado el desarrollo de sistemas de visualización, que faciliten su análisis, interpretación y manipulación. Entre estos, aquellos que cuentan con características inmersivas son de gran interés, porque permiten mayores capacidades de interacción a través de interfaces cada vez más naturales y ergonómicas, ampliando el rango de acción del análisis de información hacia aplicaciones en arte, entretenimiento y educación. Entre estos sistemas de visualización podemos enumerar los cascos de realidad virtual HMD (Head-Mounted Display); monitores binoculares omnidireccionales BOOM (Binocular OmniOriented Monitors), mesas inmersivas (ImmersaDesk) y sistemas de caverna de inmersión CAVE (Cave Automatic Virtual Environment)[2].

Un CAVE es un sistema de visualización que construye una escena a partir de proyecciones independientes desde diferentes ángulos, sobre las paredes de una habitación pequeña. Adicionalmente posee un sistema de audio envolvente y visualización estereoscópica que permite al usuario experimentar una sensación de inmersión en un ambiente virtual. La arquitectura general de un CAVE fue presentada por primera vez en 1991, en el Laboratorio de Visualización Electrónica (EVL, por sus siglas en inglés), por Thomas DeFanti y Dan Sandinen, como ambiente ideales de inmersión virtual. Un año después Carolina Cruz-Neira y otros estudiantes del EVL, bajo la dirección de DeFanti y Sandinen, realizaron la primera implementación de este sistema [2][3].

La Figura 1 presenta un esquema de la arquitectura CAVE de DeFanti y Sandinen, la cual ésta determinada por una caja cúbica de entre 7 a 10 pies de lado. Sobre tres de sus paredes (frontal y laterales) y el piso se realizan proyecciones estéreo independientes de la misma escena, ampliadas por un sistema óptico a base de espejos. El sistema de espejos tiene la finalidad de ampliar la imagen del proyector correspondiente, para reducir los requerimientos de espacio, además de lograr la proyección en ángulos difíciles como el del piso. La proyección se realiza a través de proyectores estereoscópicos que alternan las imágenes para cada ojo de manera sincronizada, por lo que el usuario debe usar gafas de cristal estéreo (shutter glasses) que separen las imágenes. El CAVE cuenta con un sistema de rastreo, que permite la interacción en tiempo real y está conformado por un conjunto de sensores ubicados en las gafas (para la posición de la cabeza) y en la mano, 
además de sistema de audio envolvente. El sistema es controlado a partir de un conjunto de equipos de cómputo de alto desempeño [2][3]. En la primera exhibición del CAVE del EVL en 1992 se usaron proyectores estéreo de una resolución de 1280 x 512 píxeles y una velocidad de refresco $120 \mathrm{~Hz}$, cuatro estaciones de trabajo Silicon Graphics comunicados y sincronizados por una red de comunicaciones de fibra óptica [2][3].

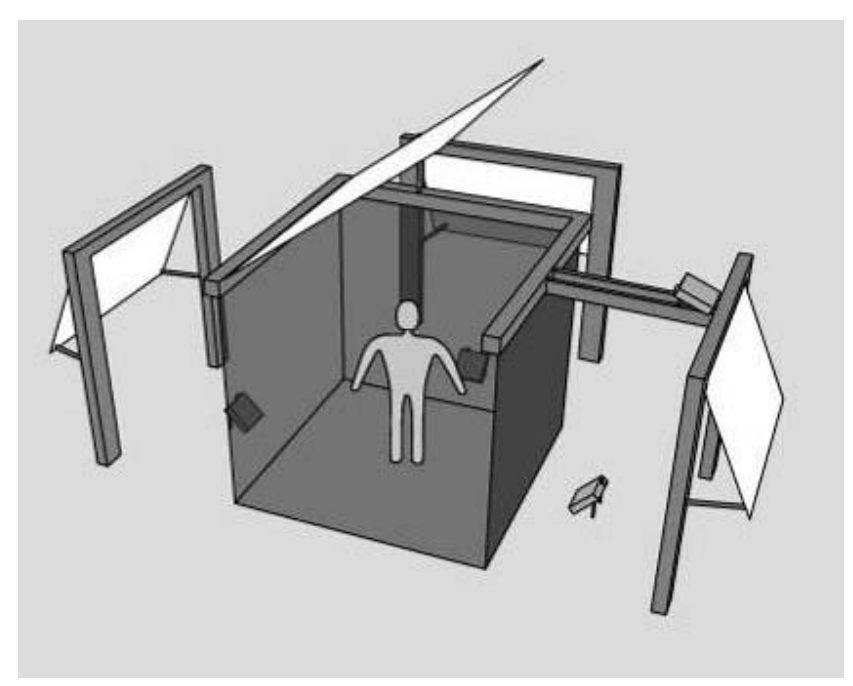

Figura 1. Esquema del CAVE propuesto por DeFanti y Sandin; se observa que se realiza proyección sobre las paredes laterales, frontal y el piso, haciendo uso de espejos.

Las aplicaciones e investigaciones que actualmente se realizan a partir de sistemas de visualización CAVE son diversas. Por ejemplo, el CAVE del NCSA (National Center for Supercomputing Applications at the University of Illinois) es usado para la visualización de datos arrojados por fenómenos naturales, visualización de sistemas caóticos, sistemas moleculares, etc.; en proyectos realizados en colaboración con la División de Matemáticas y Ciencias de la Computación del Laboratorio Argonne, el Centro de Astrofísica Termonuclear de la Universidad de Chicago y el EVL entre otros [7][20]. En la Universidad de Pittsburgh, a través del Centro Médico de Realidad Virtual se investigan los factores de control de postura en personas con y sin disfunción del balance y exploran nuevas formas de tratar esta patología médica, haciendo uso de sistemas CAVE [10]. En el Laboratorio de Investigación en Visualización de la Universidad de Brown, en colaboración de la Escuela de Diseño de Rhode Island, se está poniendo un entorno CAVE al alcance de artistas gráficos para la creación de obras digitales [13]. Los CAVE también han sido usados para recorridos virtuales, es el caso del teatro de la tierra, el cual es un sistema de inmersión virtual en el Museo de Historia Natural de Carnegie sobre el CAVEUT desarrollado en la universidad de Pittsburgh [11][12]. Así mismo, los CAVE son usados en aplicaciones de entrenamiento de personal como las desarrolladas por el Instituto de Tecnologías Creativas de la Universidad de Sur de California [17] y el Departamento de 
Ciencias de Información de la Universidad de Pittsburg [12], Los CAVE han incursionado en el entretenimiento digital, por ejemplo Paul Rajlich, investigador programador del NCSA desarrolló una modificación del conocido juego Quake II para ser corrido en un ambiente CAVE y Jeffrey Jacobson del Departamento de Ciencias de Información de la Universidad de Pittsburgh ha liderado proyectos que han realizado modificaciones al popular juego Unreal Tournament para ser ejecutado en diferentes ambientes inmersivos [11][13].

En general los laboratorios mencionados han construido su propios sistemas CAVE, pero los requerimientos técnicos del montaje (proyectores estéreo, sistemas ópticos, potentes estaciones de trabajo y redes de alta velocidad) los hacen muy costosos.

Algunos de ellos han hecho modificaciones que simplifican el sistema CAVE original, enfocándose principalmente en reducir los costos. Es así, como el Departamento de Ciencias de Información de la Universidad de Pittsburgh ha implementado algunas modificaciones del CAVE usando computadores personales, proyectores, redes de datos convencionales y algunos casos telones ensamblados a mano, con lo cual lograron reducir de manera significativa los costos. EI CAVEUT es uno de estos proyectos, fue usado para la realización del teatro de la tierra y consta de tres paredes (la frontal del doble tamaño de las laterales) y cuatro proyecciones [10]; de manera similar el sistema MiniCAVE, usa tres proyecciones que forman ángulos de visión de 60 [10], y el V-CAVE, es una versión simple que usa dos pantallas a noventa grados [14]. Otros laboratorios han realizado montajes similares, el Colegio de Computación del Instituto Tecnológico de Georgia, diseño un sistema similar al MiniCAVE, denominado The NAVE [10] y el Instituto Fraunhofer, la Universidad Técnica de Berlín y el Instituto Técnico Omikron, desarrollaron el X-Room como una alternativa CAVE de bajo costo, usando computadores personales convencionales con configuraciones de sistema de 5 ó 2 pantallas [8].

En Latinoamérica el Laboratorio de Sistemas Integrados (LSI) en la Universidad de Sao Paulo, Brasil, inició con una validación de un sistema de bajo costo [21], logrando inaugurar en el 2001 un CAVE de 5 pantallas y cuentan hoy con proyectos de investigación culminados y en desarrollo principalmente en ingenierías naval, oceánica y de automóviles [22] y recorridos virtuales de arquitectura [23]. Así mismo, el laboratorio NTAV (Novas Tecnologias nas Artes Visuais) de la Universidad de Caxias do Sul, están realizando aplicaciones artísticas en un sistema de inmersión virtual [4]. En nuestro país la facultad de ingeniería de la Universidad de los Andes publicó en el 2004 que planeaba la construcción del laboratorio de visualización y telecolaboración [6],[16], el cual se encuentra en construcción e incluirá algunos conceptos de los sistemas CAVE [5], además, en los últimos años han dirigido proyectos de fin de carrera y tesis de maestría que apuntan a la construcción de una librería para una plataforma CAVE y el desarrollo de aplicaciones creadas con diferentes herramientas $[1,6,16]$.

Este artículo presenta el diseño de un prototipo de sistema de realidad virtual inmersivo CAVE simplificado de dos pantallas ubicadas a $90^{\circ}$, se incluye el diseño de un volumen de vista 3D para dicho sistema y su validación mediante la construcción de prototipos de 
bajo costo. El objetivo es mostrar la capacidad de implementación de sistemas inmersivos, en laboratorios con capacidades limitadas de cómputo. Esto con el fin de promover el uso de estas tecnologías en aplicaciones de alto impacto. El artículo se divide de la siguiente manera: la primera sección presenta los conceptos generales de un sistema de visión 3D; la segunda presenta el diseño del volumen de vista 3D, la arquitectura del sistema inmersivo y resultados logrados, por último se presentan las conclusiones y perspectivas de trabajo futuro.

\section{MARCO CONCEPTUAL}

\subsection{VENTANA DE VISTA Y PUERTO DE VISTA}

La ventana de vista es una sección de una escena 2D que se desea graficar en una sección del dispositivo de graficación llamado puerto de vista. Generalmente tanto el puerto de vista como la ventana de graficación son rectángulos paralelos a los ejes de coordenadas y están limitados por valores máximos y mínimos como se muestra en la Figura 2.

(a)

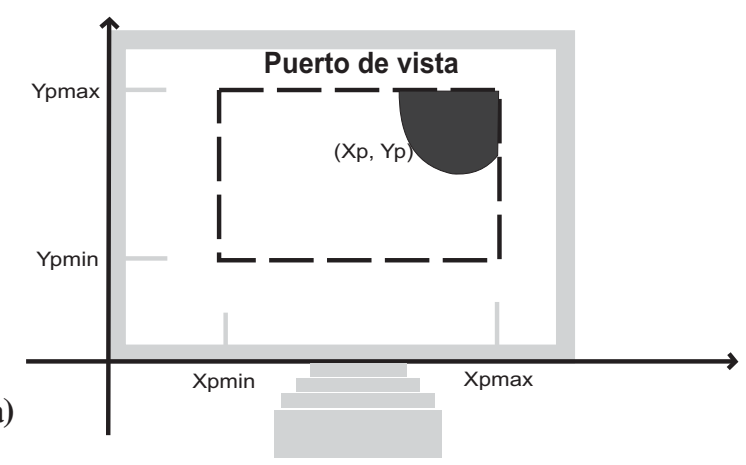

(b)

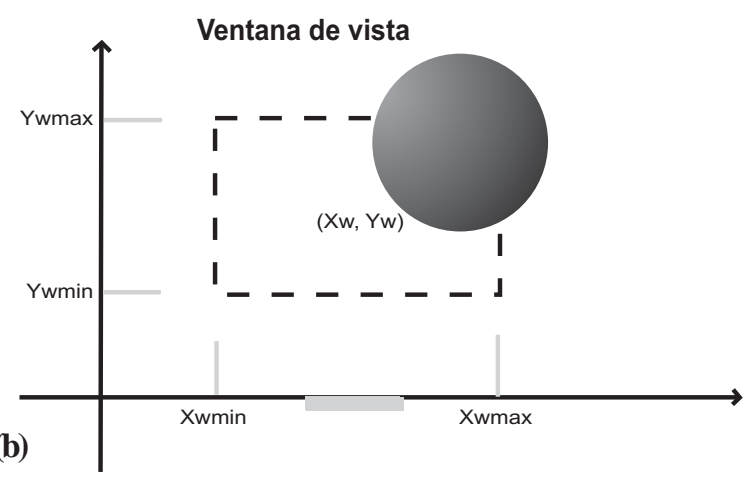

Figura 2. Ventana de graficación, (a) Puerto de vista, (b) Ventana de vista.

\subsection{VOLUMEN DE VISTA}

La generalización de una ventana de vista en 3D se denomina volumen de vista. Este se encuentra orientado para observar una sección de una escena 3D, es decir, se define una cámara con la cual se va a visualizar la escena y su propio sistema de coordenadas denominado coordenadas de vista $(v)$. Definiremos este sistema con origen en $\vec{O}_{v}$ y sus ejes orientados sobre los vectores unitarios $\vec{u}, \vec{v}, \vec{n}$, donde $\vec{n}$ indica el vector normal a la cámara. El volumen de vista se encuentra limitado por los semiplanos cercano y lejano respectivamente. Para graficar todo punto dentro del volumen de vista, esté debe ser proyectado sobre el plano de proyección ubicado sobre $z_{p}$. 
(a)

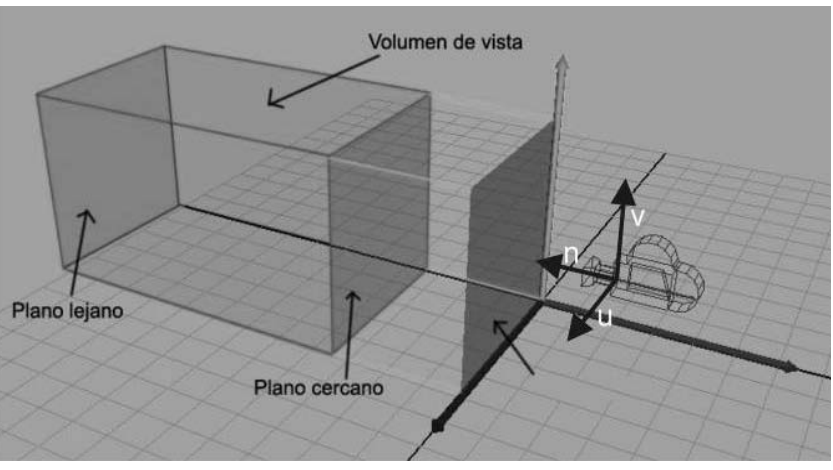

(b)

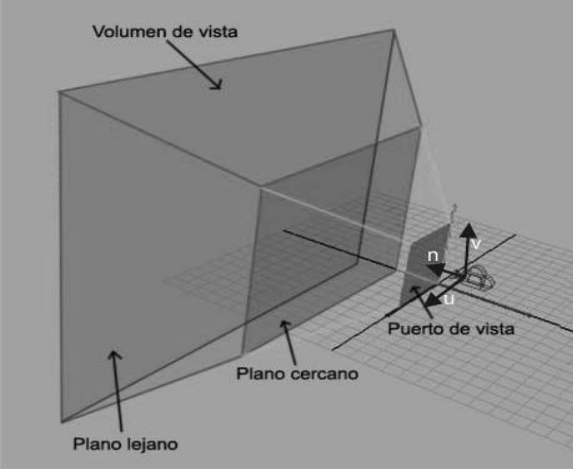

Figura 3. (a) Un volumen de vista proyección paralela. (b) Un volumen de vista proyección perspectiva.

La transformación de proyección usada establece la forma del volumen de vista, una proyección paralela define un volumen de vista como paralelepípedo rectangular (Figura 3(a)); y en una proyección perspectiva el volumen de vista es una pirámide truncada en el plano cercano (Figura 3(b)).

\subsection{VOLUMEN DE VISTA INMERSIVO}

En un sistema de inmersión tipo CAVE se establecen volúmenes de vista independientes y complementarios de un mismo campo visual para un único punto focal, ubicado en la posición del usuario. Un sistema CAVE posee dispositivos de rastreo de posición, por lo tanto el punto focal es actualizado con la posición del usuario. La Figura 4 muestra un ejemplo de un volumen de vista inmersivo para tres pantallas.

(a)

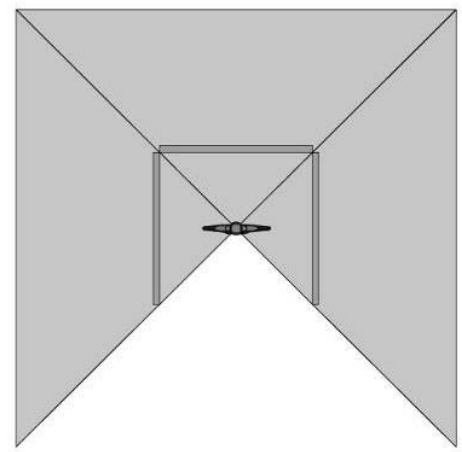

(b)

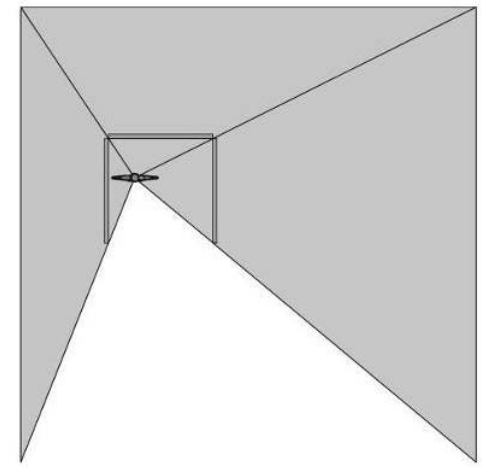

Figura 4. Volumen de vista inmersivo para un sistema de tres pantallas. Se observa que el volumen está compuesto por tres volúmenes de vista independientes con punto focal ubicado en posición del usuario. En (a) se observan los volúmenes de vista cuando el usuario está ubicado a la misma distancia de las tres pantallas, en (b) se muestra cómo los volúmenes de vista cambian de acuerdo a la posición del usuario, esto se logra con la información arrojada por el dispositivo de rastreo de posición. 


\section{MATERIALES Y MÉTODOS}

\subsection{DISEÑO DEL VOLUMEN DE VISTA INMERSIVO}

El sistema de inmersión simplificado propuesto está formado por dos pantallas no estereográficas a $90^{\circ}$ en "V", conocido como V-CAVE [14][18], similar a las propuestas del laboratorio de visualización de la Universidad de Pittsburg y del instituto Fraunhofer [14][8]. Además, se eliminó el sistema de rastreo de posición, lo que establece el punto focal en un punto fijo llamado punto ideal de visión. Dos esquemas de de proyección fueron evaluados para la implementación del volumen de vista inmersivo: frontal ó trasera.

La proyección frontal, mostrada en los diagramas (b) y (d) de la Figura 5 permite un mayor tamaño de proyección con el mismo requerimiento de espacio que la proyección trasera. Sin embargo, presenta un área de interacción más reducida, que impide al usuario acercarse a las pantallas más allá del cono de proyección, donde eventualmente su sombra interferirá con la imagen desplegada. Esto implica que el punto ideal de visión debe ser ubicado justo en el punto donde el usuario logre un máximo acercamiento a las pantallas, restringiendo el ángulo de visión a $a_{v}$ el cual es siempre menor de $180^{\circ}$. Este esquema requiere de proyectores con corrección de trapecio horizontal, características que la mayoría de estos equipos no poseen y aumenta el costo del mismo.

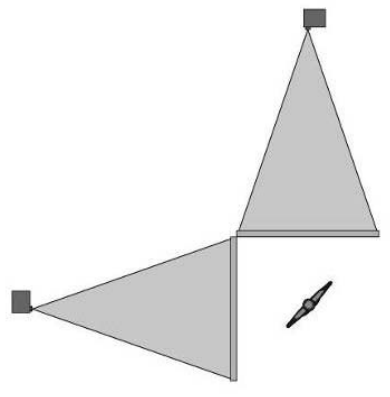

(a)

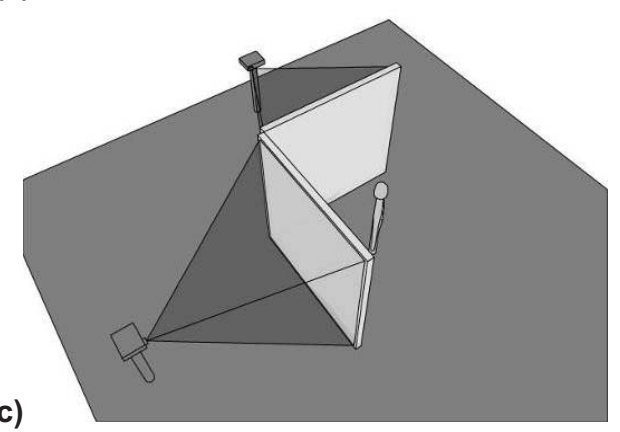

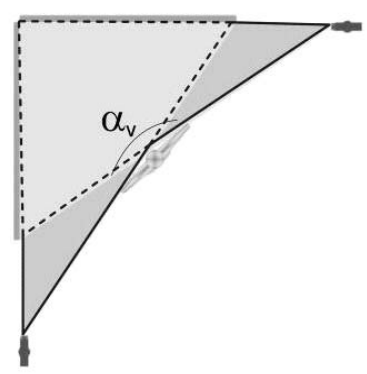

(b)

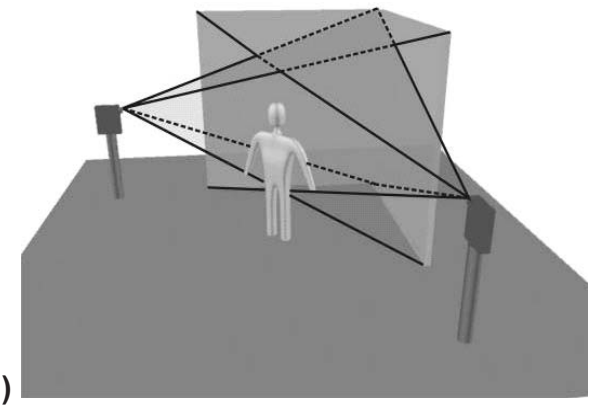

Figura 5. Diagramas de proyección. Proyección trasera: (a) y (c) vista superior y perspectiva. Proyección delantera: (b) y (d) vista superior y perspectiva, respectivamente. 
La proyección trasera, diagramas (a) y (c) de la Figura 5, necesita un mayor requerimiento de espacio para lograr la misma área de proyección. Sin embargo, presenta una completa zona de interacción dentro del sistema, permitiendo que el usuario se acerque a las pantallas tanto como lo desee. Otro atractivo de este esquema, es que los proyectores a ser usados no requieren ningún tipo de corrección de trapecio, si estos se encuentran perfectamente alineados con respecto al centro de las pantallas. Es claro que el anterior supuesto es difícil de cumplir en la realidad, pero con una corrección de trapecio vertical la imagen puede alinearse, característica disponible en cualquier proyector. Además, en este esquema de proyección el punto ideal de visión se ubica en el centro de las dos pantallas, donde el usuario tiene un ángulo visual de $180^{\circ}$. Por todo lo anterior se eligió un esquema de proyección trasera para el volumen de vista inmersivo.

En éste esquema los volúmenes de vista independientes son simétricos con respecto a la diagonal en un ángulo de $45^{\circ}$ que pasa por la esquina de unión de las dos pantallas, y se encuentran orientados a $90^{\circ}$ uno con respecto al otro, como se muestra en la Figura 6. Además, si los volúmenes de vista guardan una proporción $W_{w} / H_{w}=4 / 3$ (actualmente sigue siendo la más usada), también se puede afirmar que la distancia focal es de $W_{w} / 2$, y por lo tanto es posible concluir que los volúmenes de vista deben tener ángulos de apertura horizontal y vertical de $90^{\circ}$ y $73^{\circ}$ respectivamente.

(a)

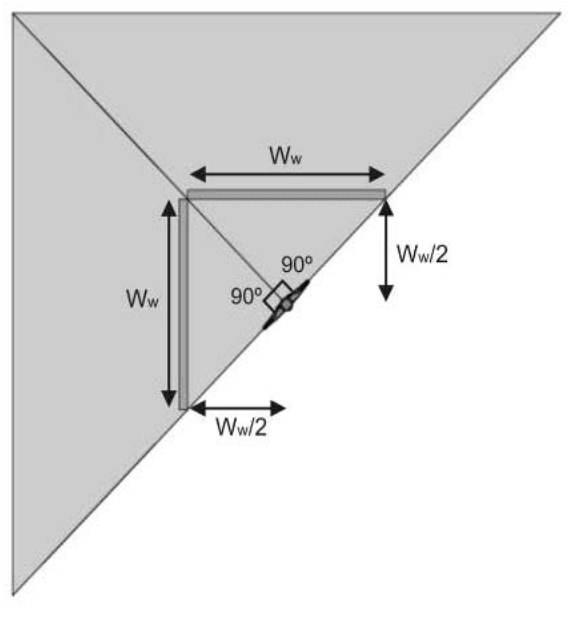

(b)

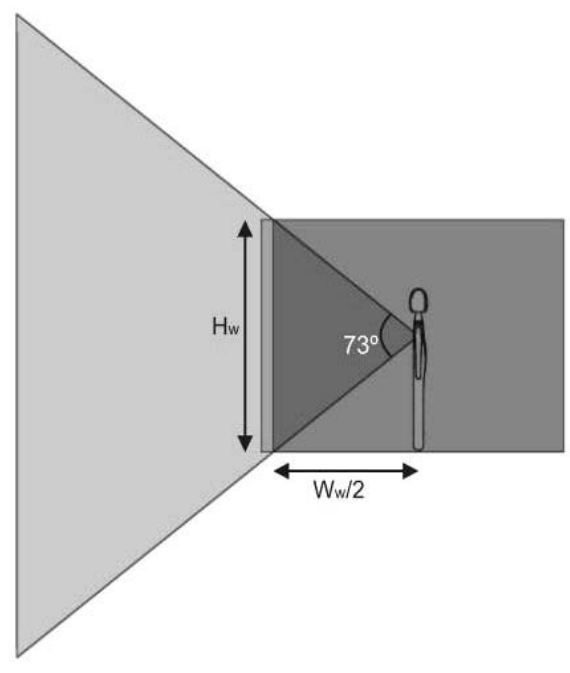

Figura 6. Volumen de vista inmersivo diseñado para un sistema simplificado de dos pantallas. (a) Vista superior. (b) Vista lateral. 


\section{2-2. ARQUITECTURA DEL SISTEMA}

Las arquitecturas de sistemas inmersivos de dos pantallas presentadas en [14][18] utilizan un total de tres equipos de cómputo, dos para la proyección y un servidor que se encarga de la sincronización de los dos anteriores, a partir de una red de datos. Dadas las características técnicas del hardware de video actual que permite dos salidas de video simultáneas e independientes con aceleración 3D sobre una sola tarjeta, se propuso una arquitectura que hiciera uso de dicha ventaja técnica, permitiendo reducir el número de equipos de cómputo a sólo uno, habilitado con una tarjeta de video de este tipo, como se muestra en la Figura 7(b).

(a)

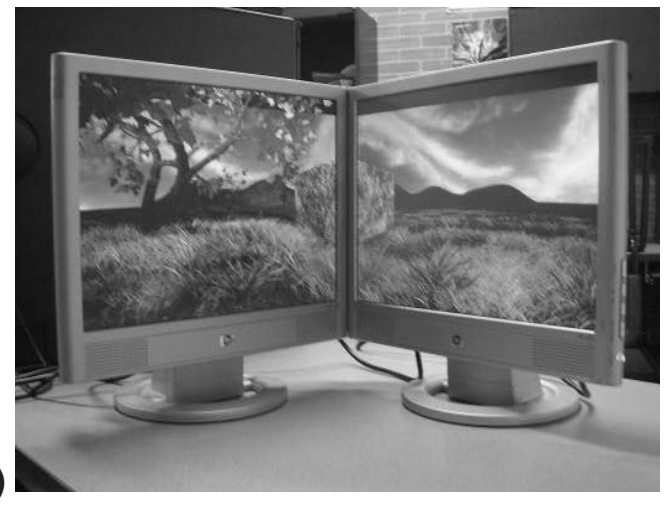

(c)

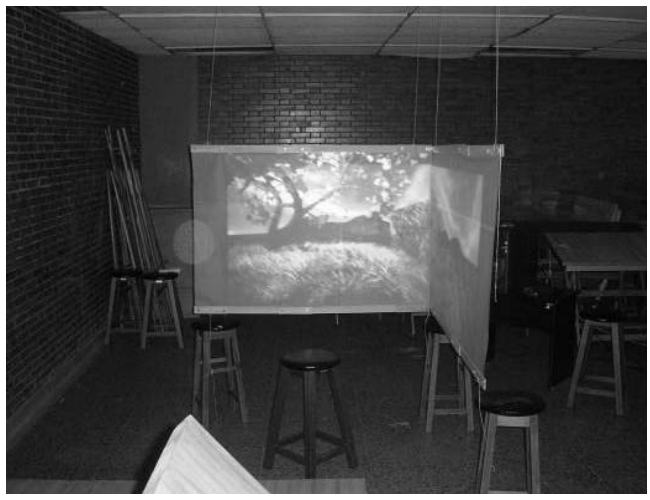

(b)

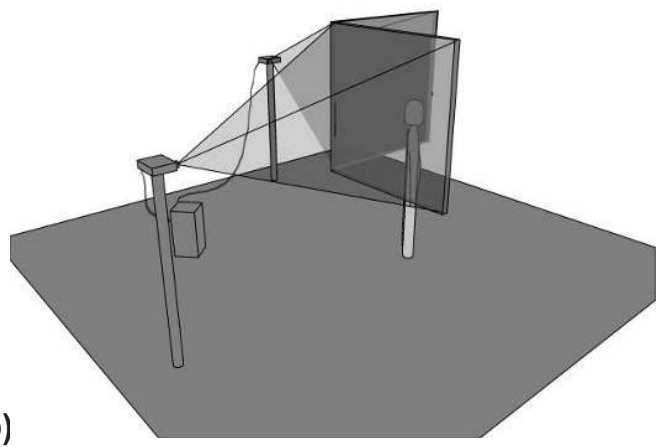

(d)

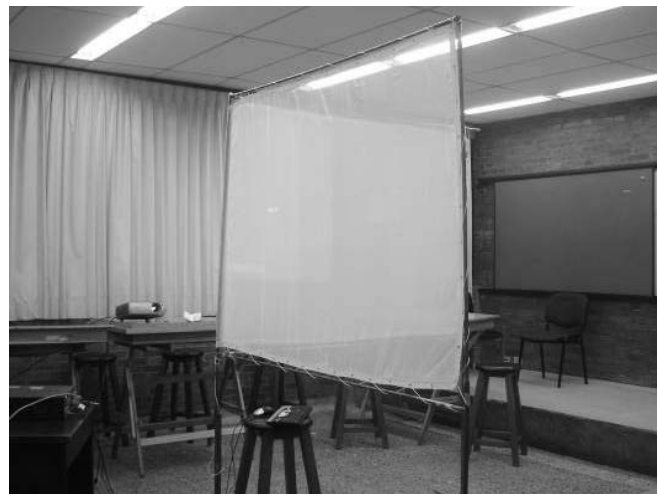

Figura 7. (a) prototipo de mesa con monitores LCD, (b) arquitectura del sistema con esquema de proyección trasera, (c) prototipo de tamaño real de estructura de perfiles de madera y telones en pergamino colgado al techo y (c) prototipo final de estructura en aluminio y telones en tela translucida. 
El sistema inmersivo aquí presentado se implementó sobre una estación de trabajo DELL Precision 630 provista con una tarjeta de video NVIDIA QUADRO4 580 XG. Las características técnicas de esta máquina se presentan en las tablas 1 y 2 . Estas características se pueden tener en la mayoría de computadores de escritorio que se encuentran en el mercado actual, lo que demuestra su implementabilidad y replicabilidad.

Tabla 1. Características técnicas de la estación de trabajo Dell Precision 630

\begin{tabular}{|l|r|}
\hline Procesador & Intel XEON, 2 GHz, L1 512 KB \\
\hline Chipset & Intel E7505 \\
\hline Disco duro & $80 \mathrm{~GB}, 7.200 \mathrm{rpm}$ \\
\hline Memoria RAM & $1 \mathrm{~GB}$ DDR, 333 MHz \\
\hline
\end{tabular}

Fuente: http://support.dell.com

Se desarrolló un primer prototipo utilizando dos monitores LCD HP vs15 ubicados sobre una mesa formando un ángulo de $90^{\circ}$. Este montaje tiene la ventaja de requerir poco espacio y facilitar la elaboración de pruebas, aunque no ofrece la experiencia de inmersión que se quiere lograr, debido al tamaño de las pantallas y el borde de los monitores. La Figura 7(a) muestra una fotografía de una escena visualizada a través de este prototipo.

Tabla 2. Características técnicas de la tarjeta gráfica Nvidia QuadrO4 580 XGL

\begin{tabular}{|l|c|}
\hline nView Multi-Display & 2 salidas \\
\hline RAM & 64 MB DDR \\
\hline BUS & AGP 8X \\
\hline Fabricación del GPU & 0.11 micron \\
\hline RAMDAC & $350 \mathrm{MHz}$ \\
\hline Triángulos por segundo & 60 '000.000 \\
\hline
\end{tabular}

Fuente: http://www.nvidia.com/page/quadro4xgl.html

Se procedió a elaborar un prototipo con sistema de proyección, para lo cual se usaron proyectores LDC Sanyo PLC-XU73 4.2, con las especificaciones técnicas de la Tabla 3. El principal problema que se presentó en la construcción de este prototipo fue el requerimiento de espacio físico, debido a que en las instalaciones no se contaba con un lugar adecuado para realizar una proyección "piso - techo". Se decidió realizar un prototipo con pantallas más pequeñas para disminuir el requerimiento de espacio, ubicándolas de tal manera que la cabeza de una persona de $170 \mathrm{~cm}$ de estatura (siendo la estatura promedio en Latinoamérica de $165 \mathrm{~cm}$ a $175 \mathrm{~cm}$ ) estuviera en el punto ideal de visión, ver Figura 7(b). 
Tabla 3. Características técnicas del proyector LDC Sanyo PLC-XU73 4.2

\begin{tabular}{|l|r|}
\hline Relación Ancho/Alto & $4 / 3$ \\
\hline Resolución máxima (pixeles) & 1024 X 768 \\
\hline lluminación con lámpara nueva(lumines) & 200 \\
\hline Máxima distancia de proyección (pies) & 43.3 \\
\hline Mínima distancia de proyección (pies) & 4.6 \\
\hline Máxima tamaño de imagen en diagonal (pulgadas) & 300 \\
\hline Mínimo tamaño de imagen en diagonal (pulgadas) & 40 \\
\hline Proyección en posición de mesa & $\mathrm{SI}$ \\
\hline Proyección en posición de techo & $\mathrm{SI}$ \\
\hline Proyección por detrás del telón & $\mathrm{SI}$ \\
\hline Corrección de trapecio vertical & $\mathrm{SI}$ \\
\hline Corrección de trapecio horizontal & $\mathrm{NO}$ \\
\hline
\end{tabular}

Fuente: http://www.sanyo-Icdp.com/english/

Para este segundo prototipo se construyeron dos telones de $120 \mathrm{~cm} \times 160 \mathrm{~cm}$ sobre perfiles de madera de $5 \mathrm{~cm}$ de ancho y papel pergamino de 120 gramos, el cual permitía una visualización translucida de la imagen proyectada. Los telones se colgaron del techo a una altura de $230 \mathrm{~cm}$, lo cual permitía al usuario estar de pie en el medio de las pantallas. El prototipo construido requirió un área de $6.60 \mathrm{~m} \times 6.60 \mathrm{~m}$, ya que los proyectores se deben ubicar como mínimo a $5 \mathrm{~m}$ de cada telón. En la Figura 7(c) se puede observar una fotografía que permite ver las características del prototipo construido.

La desventaja principal del segundo prototipo era su estructura frágil, por lo que se diseñó un tercer prototipo con una estructura en varilla de aluminio ( $3 / 4$ y $1 / 2$ de pulgada) y telones de tela translucida, guardando las dimensiones del segundo prototipo, (telones de 120 $\mathrm{cm} \times 160 \mathrm{~cm}$ ). La estructura metálica diseñada permite un ensamble y desensamble fácil y portable, ver Figura 7(d).

\subsection{ELABORACIÓN DE DATOS DE PRUEBA}

Para el software de modelado y animación 3D (3D AutoDesk Maya 7.0) se crearon dos cámaras (volúmenes de vista) de acuerdo con el volumen de vista inmersivo presentado en la sección 2.1. La configuración de las cámaras se pueden ver en la Figura 8. Se creó un conjunto de animaciones $3 \mathrm{D}$ con objetos que salían y entraban en cada uno de los volúmenes de vista. Para cada una de las animaciones se realizó un proceso de render independiente de cada cámara. La Figura 8 muestra el conjunto de las animaciones creadas, que se listan a continuación:

- Un cubo en movimiento entre las pantallas 
- Una habitación cúbica con textura de ajedrez en techo, piso y paredes, con movimiento en la textura sobre las paredes.

- Un ciclo de una escena natural

- Un recorrido por un apartamento

(a)

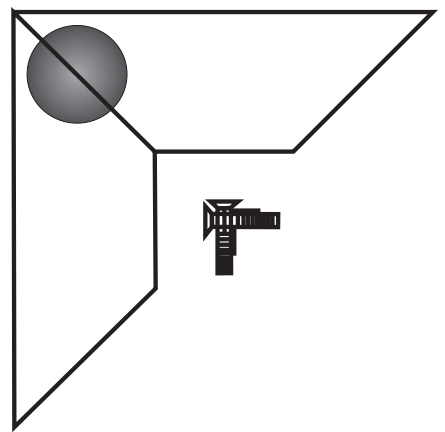

(b)

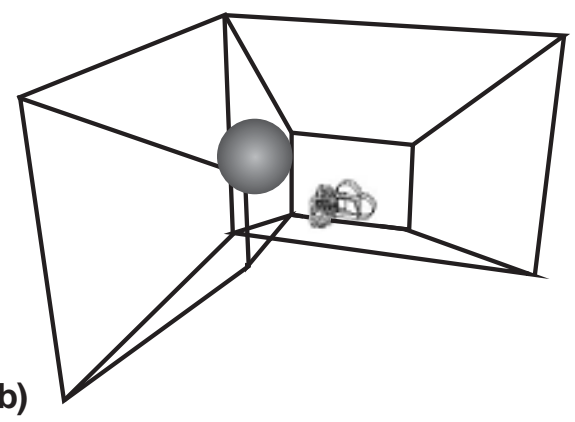

Figura 8. Configuración de la posición de las cámaras en 3D AutoDesk Maya 7.0. (a) vista superior, (b) vista perspectiva.

Para la visualización se desarrolló una aplicación que permite el despliegue de cada imagen en la respetiva salida de video de manera simultánea a la tasa de refresco adecuada para el video (30 cuadros por segundo). Lo anterior no exige requerimiento técnico alguno debido a que en esta arquitectura un solo equipo de cómputo maneja las dos salidas de video, eliminado cualquier problema de sincronismo. Se realizaron pruebas técnicas de visualización con la primera animación a resoluciones de 640 x 480, 800 x 600 y 1024 × 768, mostrando un correcto despliegue y sincronismo en todas las resoluciones. Teniendo en cuenta que actualmente NTSC y PAL siguen siendo los formatos más comunes en aplicaciones de video, se decidió realizar el proceso de render para las animaciones restantes a $800 \times 600$, por ser ésta la mínima resolución nativa en un PC superior a las especificadas en dichos formatos (720 x 486 en NTSC y 768 x 576 en PAL).
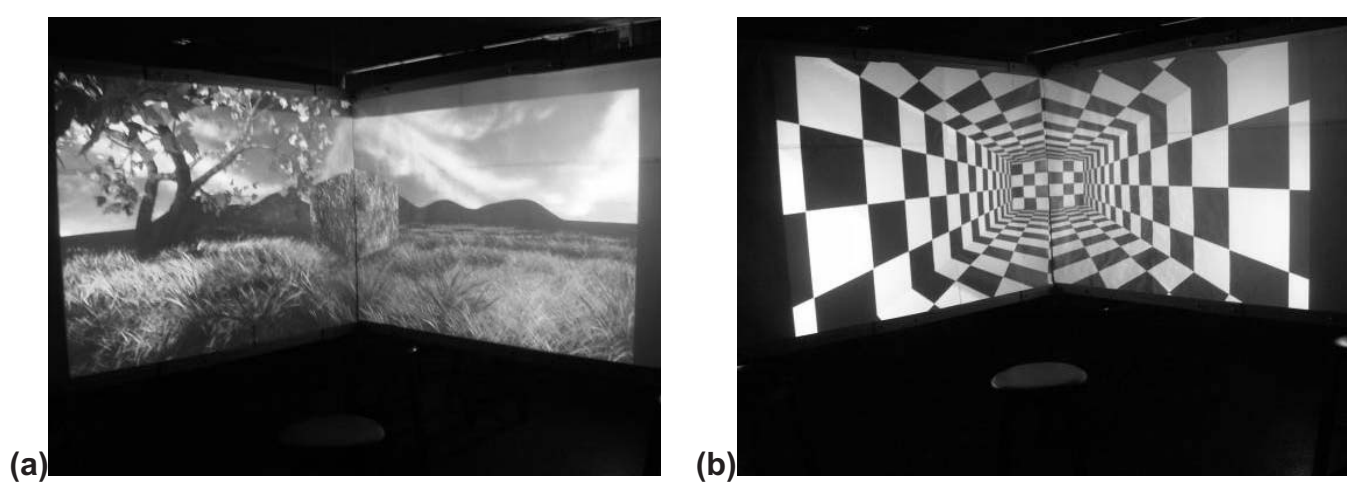

Figura 9. Fotografías de las animaciones creadas visualizadas en tercer prototipo: (a) cubo en movimiento entre las pantallas, (b) habitación cúbica con textura de ajedrez, 

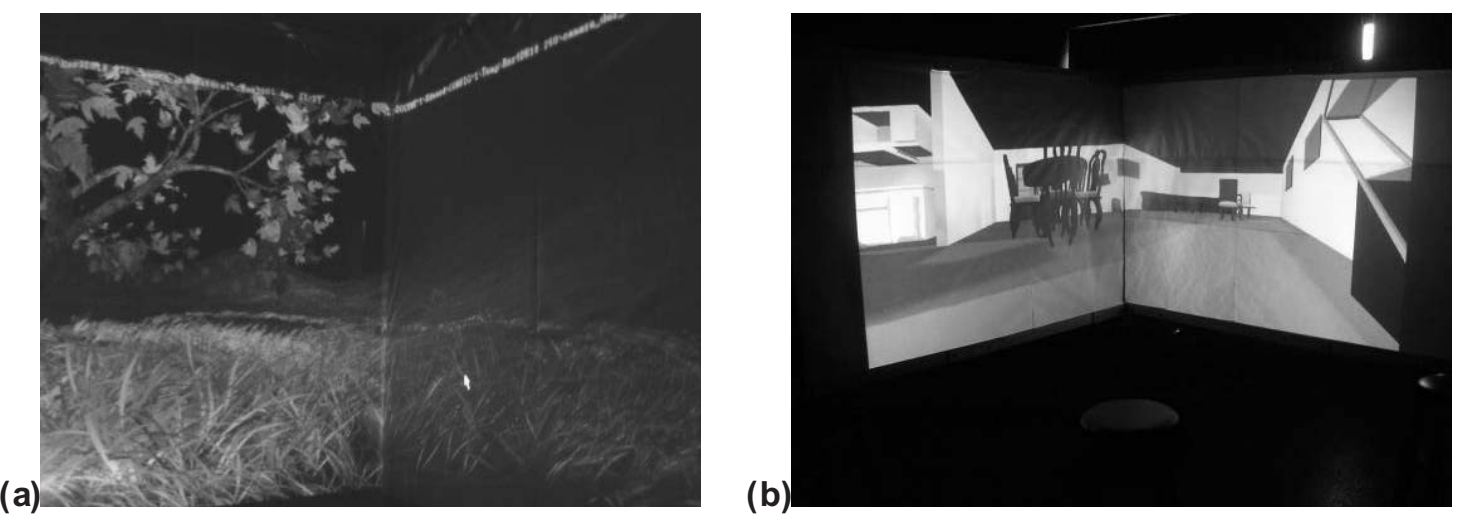

Figura 10. Fotografías de las animaciones creadas visualizadas en tercer prototipo: (a) ciclo de una escena natural y (b) recorrido por un apartamento.

\section{CONCLUSIONES Y PERSPECTIVAS}

Se presentó el diseño de un volumen inmersivo de dos pantallas y su validación en prototipos de bajo costo. Se demuestra que la implementación de este tipo de sistemas es posible con un costo inferior a los 20 millones de pesos colombianos. Sin embargo, vale la pena mencionar que el sistema presentado es de menor costo, debido a que se uso equipos desactualizados para mostrar la facilidad de dicha implementación.

El volumen de vista inmersivo diseñado fue validado mediante la presentación de cuatro animaciones, donde se evaluó de manera positiva tanto la coherencia visual entre los dos volúmenes de vista 3D complementarios, como la fluidez de las animaciones; lo cual permite concluir que la arquitectura propuesta es adecuada para la proyección de escenas inmersivas.

Lo anterior permite afirmar que es posible aprovechar, en el desarrollo de sistemas inmersivos de bajo costo, la característica actual que posee algunas tarjetas de video para manejar múltiples salidas independientes. Lo que reduce el costo del sistema y facilita el desarrollo de aplicaciones al eliminar el problema de sincronismo entre equipos.

Por otro lado, el prototipo final implementado logró superar el requerimiento de espacio físico con telones flotantes en una estructura portátil que permite su ensamble y desensamble, siendo un problema la puesta a punto del sistema inmersivo, debido a la necesidad de la calibración manual de las proyecciones, cada vez que se ensamble el sistema. También se resalta la versatilidad que presenta el prototipo de escritorio, ya que permite una rápida puesta a punto y bajo requerimiento de espacio en la realización de todas las pruebas, sin necesidad de armar el montaje de tamaño real, el cual se ensambla cuando los resultados son adecuados. 
Con el último prototipo presentado se inició una línea de investigación que pretende desarrollar nuevos prototipos inmersivos, aplicaciones para estos dispositivos e investigaciones encaminada a la solución de problemas en el contexto tanto local como mundial. Actualmente se está diseñando un prototipo con más pantallas, se están desarrollando diferentes aplicaciones inmersivas y un sistema de calibración automática de proyecciones por medio de modelos algorítmicos y matemáticos de visión por computador, para solucionar el problema de puesta a punto en prototipos portátiles de sistemas inmersivos.

\section{REFERENCIAS BIBLIOGRÁFICAS}

[1] CHAVARRO GARCÍA, Oscar Javier, (2001), Plataforma para el desarrollo de aplicaciones de realidad virtual Aquynza. Proyecto final de Magister en Ingeniero Sistemas y Computación, Universidad de los Andes, Facultad de Ingeniería, Departamento de Ingeniería de Sistemas y Computación.

[2] CRUZ NEIRA, C., SANDIN, D., DEFANTI, T., KENYON, R. y HART, J.; (1992), The CAVE: Surround-Screen Projection-Based Virtual Reality: The Design and Implementation of the CAVE. En: ACM Press, pp. 135 - 142.

[3] CRUZ NEIRA, C., SANDIN, D., DEFANTI, T.; (1993), The CAVE: Audio Visual Experience Automatic Virtual Environment. En: Communications of the ACM, pp. 6572.

[4] DOMINGUES, Diana; MORAES, Mauricio; Conexão - comunicação e cultura: ciberespaço, comunicação e criação, (2005), Realidade virtual e a imersão em caves. En: Revista de Comunicação da Universidade de Caxias do Sul, Vol. 3, No 6.

[5] FIGUEROA, Pablo, HERNÁNDEZ, José Tiberio; (2004), Infraestructura de visualización y telecolaboración para la nueva facultad de ingeniería. En: revista de ingeniería, facultad de ingeniería universidad de los andes, № 04, pp. 84-89.

[6] FUENTES HERRERA, Omar Alejandro, (2001), Esquema de distribución para ambientes virtuales multi-usuario. Proyecto final de Magister en Ingeniero Sistemas y Computación, Universidad de los Andes, Facultad de Ingeniería, Departamento de Ingeniería de Sistemas y Computación.

[7] HUDSON, Randy, GUNN, Charlie, FRANCIS, George K., SANDIN, Daniel J., DEFANTI, Thomas; (1995), The Mathenautics: Using VR to Visit 3-D Manifolds Randy Hudson. En: ACM Press, pp. 167 - 170. 
[8] HEARN, Donald, BAKER, M. Pauline, (1997). Computer Graphics. Prentice Hall, pp $453-478$.

[9] ISAKOVIC, Karsten, DUDZIAK, Thomas, KOCHY, Kai; (2002), X-Rooms APC-based immersive visualization environment. En: ACM Press, pp. 173 - 177.

[10] JACOBSON, Jeffrey, REDFERN, Mark S., FURMAN, Joseph M., WHITNEY, Susan L., SPARTO, Patrick J., WILSON, Jeffrey B., HODGES, Larry F.; (2001), Balance NAVE; A Virtual Reality Facility for Research and Rehabilitation of Balance Disorders. En: ACM Press, pp. 103 - 109.

[11] JACOBSON, Jeffrey; (2002), Configuring Multiscreen Displays With Existing Computer Equipment. En: Human Factors and Ergonomics Society 46th Annual Meeting.

[12] JACOBSON, Jeffrey, LEWIS, Michael; (2005), Game engine virtual reality with CAVEUT. En: IEEE Computer, Vol 38, pp. $79-82$.

[13] JACOBSON, Jeffrey, LE RENARD, Marc, LUGRIN, Jean-Luc, CAVAZZA, Marc; (2005), The CaveUT System: Immersive Entertainment Based on a Game Engine. En: ACM Press, pp. 184 - 187.

[14] JACOBSON, Jeffrey, HWANG, Zimmy; (2002), Unreal tournament for immersive interactive theater. En: Communications of the ACM, Vol. 45, pp 39-42.

[15] KEEFE, Daniel F., ACEVEDO FELIZ, Daniel, MOSCOVICH, Tomer, LAIDLAW, David H., LAVIOLA JR., Joseph J.; (2001), CavePainting: A Fully Immersive 3D Artistic Medium and Interactive Experience. En: ACM Press, pp. 85 - 93.

[16] MEJÍA PADILLA, Daniel Felipe, (2004), Infraestructura multiplataforma para el desarrollo de aplicaciones de realidad virtual. Proyecto final de Magister en Ingeniero Sistemas, Universidad de los Andes, Facultad de Ingeniería, Departamento de Ingeniería de Sistemas y Computación.

[17] PAIR, Jarrell, PIEPO, Diane; (2002), Flatworld: A mixed reality environment for education and training. En: International Conference on Information Systems.

[18] SAUTER, P. M., (2003), VR2Go A New Method for Virtual Reality Development. En: Computer Graphics, Vol. 37, pp $19-24$.

[19] SEAY, A.F., KRUM, D.M., HODGES, L., RIBARSKY, W. (2001), Simulator sickness and presence in a high FOV virtual environment. En: IEEE, pp. 299 - 300. 
[20] TUFO, H. M., FISCHER, P. F., PAPKA, M. E., BLOM, K.; (1999), Numerical Simulation and Immersive Visualization of Hairpin Vortices. En: ACM Press, pp. 62.

[21] ZUFFO, João Antonio, SOARES Luciano Pereira, ZUFFO Marcelo Knörich, LOPES Roseli de Deus; (2001), CAVERNA Digital - Sistema de Multiprojeção Estereoscópico Baseado em Aglomerados de PCs para Aplicações Imersivas em Realidade Virtual. En: IV Symposium on Virtual and Augmented Reality.

[22] ZUFFO. M. K.; (2004), An X3D Browser for VR Immersive Simulation Based on Clusters of Commodity Computers. En: Web3D 2004 Symposium, Monterrey.

[23] ZUFFO. M. K.; (2004), Virtual Hang-gliding over Rio de Janeiro. En: IEEE VR 2004. 